IMECE2011-62533

\title{
DEVELOPMENT OF A PRACTICAL BROADBAND ACTIVE VIBRATION CONTROL SYSTEM
}

\author{
Noah H. Schiller \\ NASA Langley Research Center \\ Hampton, Virginia, USA
}

\author{
Daniel F. Perey \\ NASA Langley Research Center \\ Hampton, Virginia, USA
}

\author{
Randolph H. Cabell \\ NASA Langley Research Center \\ Hampton, Virginia, USA
}

\begin{abstract}
The goal of this work is to develop robust, lightweight, and low-power control units that can be used to suppress structural vibration in flexible aerospace structures. In particular, this paper focuses on active damping, which is implemented using compact decentralized control units distributed over the structure. Each control unit consists of a diamond-shaped piezoelectric patch actuator, three miniature accelerometers, and analog electronics. The responses from the accelerometers are added together and then integrated to give a signal proportional to velocity. The signal is then inverted, amplified, and applied to the actuator, which generates a control force that is out of phase with the measured velocity.

This paper describes the development of the control system, including a detailed description of the control and power electronics. The paper also presents experimental results acquired on a Plexiglas window blank. Five identical control units installed around the perimeter of the window achieved $10 \mathrm{~dB}$ peak reductions and a $2.4 \mathrm{~dB}$ integrated reduction of the spatially averaged velocity of the window between 500 and $3000 \mathrm{~Hz}$.
\end{abstract}

\section{INTRODUCTION}

The goal of this work is to develop a practical vibration control system that can be used to reduce interior noise levels in helicopters with less weight than conventional treatment. This has the potential to improve the environment for passengers and crew, as well as improve vehicle efficiency as a result of weight savings.

A helicopter powertrain generates high-frequency mechanical loads, which propagate throughout the primary structure. These loads cause vibrations in the sidewall and windows, which then radiate sound into the cabin. The noise is often tonal in nature with strong tones between $500 \mathrm{~Hz}$ and $3 \mathrm{kHz}$. In this frequency range, the vibratory response of the structure is typically controlled by structural damping. As a result, manufactures often add constrained layer damping to the sidewall. This treatment is not typically applied to the windows, since they need to be transparent. Previous work has demonstrated the benefits of embedding transparent viscoelastic material between layers of Plexiglas [1]. With a weight increase of just $10 \%$ over the weight of the bare window, a broadband reduction of the radiated sound power from the window was achieved. The goal of this work is to develop a simple and robust active vibration control system to augment the inherent structural damping from $500 \mathrm{~Hz}$ to $3 \mathrm{kHz}$ with less than $10 \%$ added weight relative to the bare window.

The approach is to use small independent control units installed around the perimeter of a window. Each control unit implements direct velocity feedback. As the name implies, the control force is calculated by feeding back a signal proportional to velocity. Since the control force is proportional to velocity, the approach is commonly called active damping. To achieve broadband benefits, the transducers (i.e. actuators and sensors) used in the control system must be substantially matched. This means that the actuator and sensor have to couple to the structure in the same way. For instance, a collocated point force actuator and point sensor constitute a matched transducer pair. Unfortunately real transducer pairs are never perfectly matched, which limits the performance of the control system.

Gardonio and colleagues have shown that small control units, consisting of triangularly-shaped distributed actuators and point sensors, can be distributed around the perimeter of a panel to increase the structural damping [2-5]. However above approximately $1 \mathrm{kHz}$ performance is limited because of

This material is declared a work of the U.S. Government and is not subject to copyright protection in the United States. Approved for public release; distribution is unlimited. 
differences in how the actuators and sensors couple to the structural response [3].

Subsequent work by Schiller et al. [6] has shown that appropriately shaped anisotropic actuators can be used in combination with point sensors at high frequencies. However tests demonstrated that these control units are sensitive to the boundary conditions of the structure. As a result, the control units are only effective if the perimeter of the structure is clamped.

This paper describes a new diamond-shaped Macro-Fiber Composite (MFC) actuator that can be used to create a broadband active vibration control system that is not sensitive to the boundary conditions of the structure. After describing the development of the new actuator, the control electronics are discussed. Since the overall size and weight of the control system is dominated by the electronics, the circuit design is critical to the development of a practical system. The final section of this paper discusses system performance, which was assessed experimentally on a Plexiglas window.

\section{DISTRIBUTED ACTUATORS}

Generalized functions, as described by Sullivan et al. [7], can be used to describe how distributed actuators couple to a structure. For instance triangularly shaped actuators, such as the ones used in previous studies [2-6], can be modeled as a collection of point loads and line moments as depicted in Fig. 1. In this case, the moment excitation along the base edge is defined as [2]

$$
m_{b}(t)=\left(h_{s} / 2\right)\left(e_{31}\right) v_{c}(t)
$$

where $h_{s}$ is the combined thickness of the panel and the piezoelectric patch, $e_{31}$ is a piezoelectric material constant relating the electric field applied in the 3-direction to stress induced in the 1-direction, and $v_{c}(t)$ is the applied voltage. Similarly the moment excitation along the lateral edges is defined as

$$
m_{l}(t)=\left(h_{s} / 2\right)\left(m^{2} e_{31}+e_{32}\right) v_{c}(t)
$$

where $m=b /(2 a)$ is the slope of the lateral edge, $b$ is the base of the triangle, and $a$ is its height. The point forces depicted in Fig. 1 are defined as

$$
f(t)=4 m\left(h_{s} / 2\right)\left(e_{31}\right) v_{c}(t)
$$

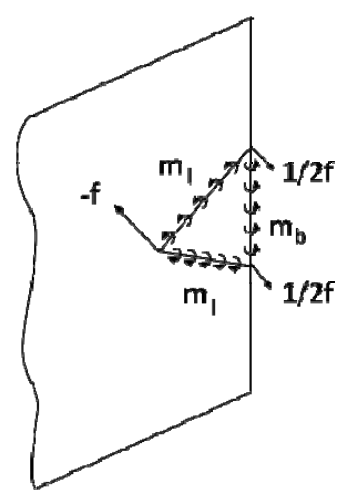

Figure 1: Triangular actuator represented in terms of point forces and line moments.

If the triangular actuator is mounted along a clamped boundary, then the point forces and line moments along the base of the actuator will not couple to the structure. Therefore a single point sensor placed at the vertex opposite the base edge will couple to the flexural response of the structure in much the same way as the actuator. However as the frequency increases and the bending wavelength approaches the dimensions of the actuator, the moments along the lateral edges couple more efficiently to the structural response than the point force at the tip of the actuator. As a result, the actuator and point sensor are not matched at high frequencies, which can limit the performance of the control system [3]. Therefore it is advantageous to eliminate the destabilizing line moments along the lateral edges of the actuator. Unfortunately this cannot be accomplished using conventional piezoelectric actuators.

Conventional actuators have uniform bipolar electrodes, one on each side of the wafer, which enable the application of the electric field through the thickness of the material (i.e. the 3-direction), as shown in Fig. 2 a). A positive voltage applied in the 3-direction induces equal compressive stresses in both inplane directions (i.e. the 1- and 2- directions). Therefore $e_{31}$ and $e_{32}$ are equal and the lateral edge moments defined in Eq. 2 cannot be eliminated. In contrast interdigitated electrodes, as shown in Fig. 2 b), allow the application of the electric field in a preferred in-plane direction. In this case a positive voltage applied in the 1-direction induces a tensile stress in the 1-direction and a compressive stress in the 2-direction. If the triangle is shaped such that $m=\left(-e_{12} / e_{11}\right)^{\wedge} 0.5$, then the destabilizing line moments defined by Eq. 2 equal zero.

This material is declared a work of the U.S. Government and is not subject to copyright protection in the United States. Approved for public release; distribution is unlimited. 

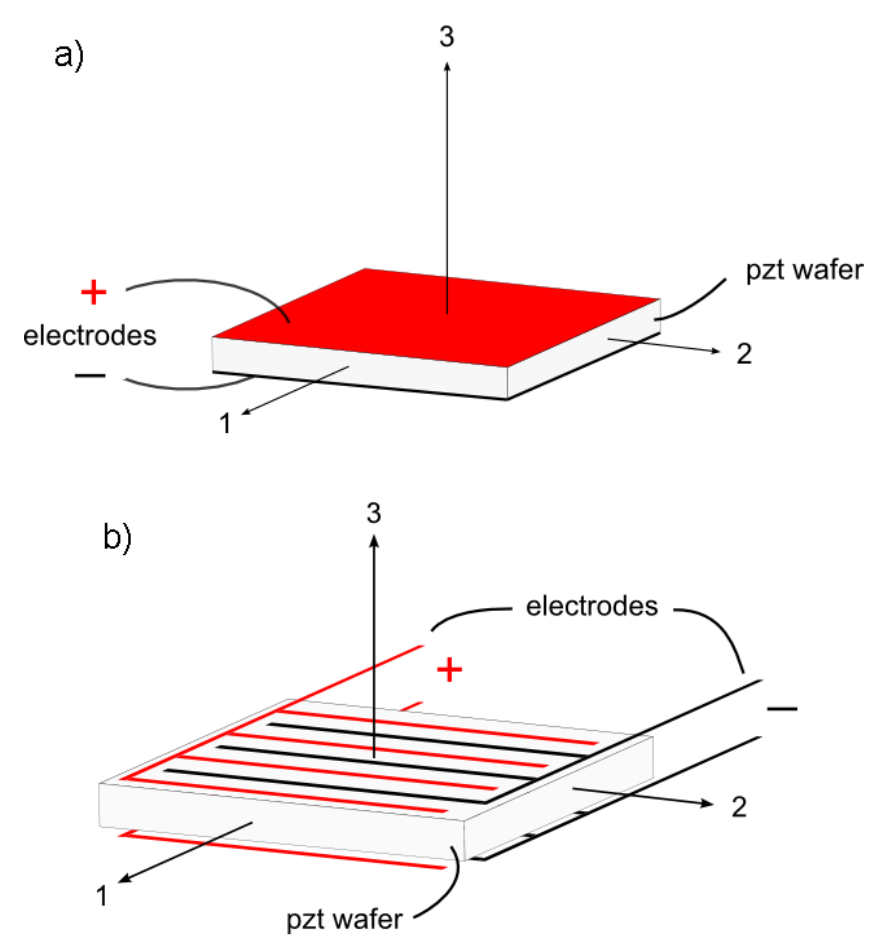

Figure 2: Sketch of (a) a conventional piezoelectric actuator, and

(b) an actuator with interdigitated electrodes. The positive

electrodes are in red and the negative electrodes are in black.

The piezoelectric material constants for a commercially available Macro-Fiber Composite (MFC) actuator with interdigitated electrodes are $e_{11}=11.9 \mathrm{~Pa} /(\mathrm{V} / \mathrm{m})$ and $e_{12}=-0.77 \mathrm{~Pa} /(\mathrm{V} / \mathrm{m})$. In this case the transducers should be designed to have a slope of $m=\left(-e_{12} / e_{11}\right)^{\wedge} 0.5=0.254$. In other words, the lateral edge moments equal zero when the height of the triangle is approximately twice the width of the base.

When mounted along a clamped boundary, this type of triangular actuator couples to the structural response in the same way as a point force. Unfortunately if the structure is not clamped, then at high frequencies the moment about the base edge couples to the structural response out-of-phase with the point force. Therefore when implemented within a control system, the base moment can destabilize the system [6].

As Fig. 3 depicts, two triangularly shaped actuators can be combined such that the moments along the base edges cancel. In addition, if the actuators have interdigitated electrodes and are shaped correctly, then there will be no lateral edge moments. The resulting diamond shaped actuator will couple to the structure in exactly the same way as four point forces. This means that a collocated transducer pair can be obtained by combining commonly available point sensors, such as accelerometers, with this type of diamond shaped actuator.

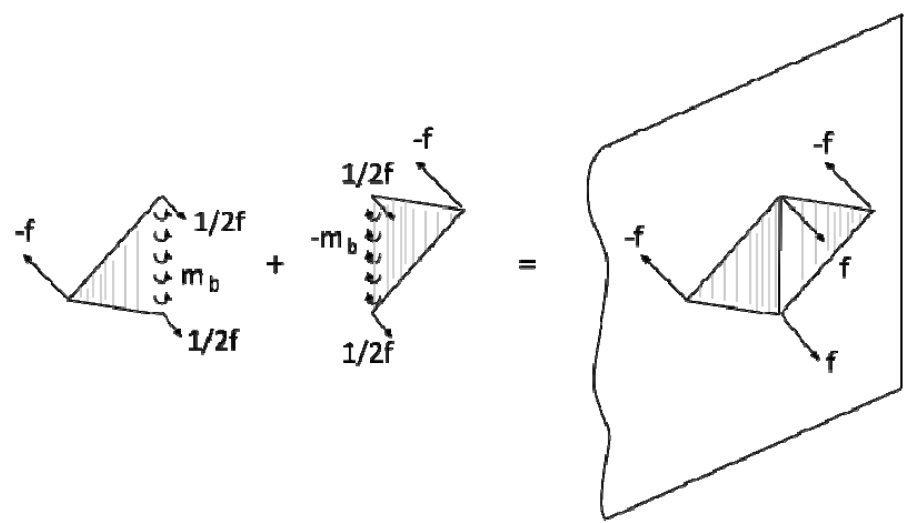

Figure 3: Optimal diamond shaped actuator created by combining two triangularly shaped actuators with interdigitated electrodes.

Since the diamond actuator couples to the structure in the same way as a collection of point forces, multiple point sensors must be combined to yield a matched sensor. Figure 4 depicts the appropriate placement and combination of sensors required to produce an equivalent sensor that is matched with the diamond shaped actuator. This type of transducer pair can be used anywhere on the structure, not just along clamped boundaries.

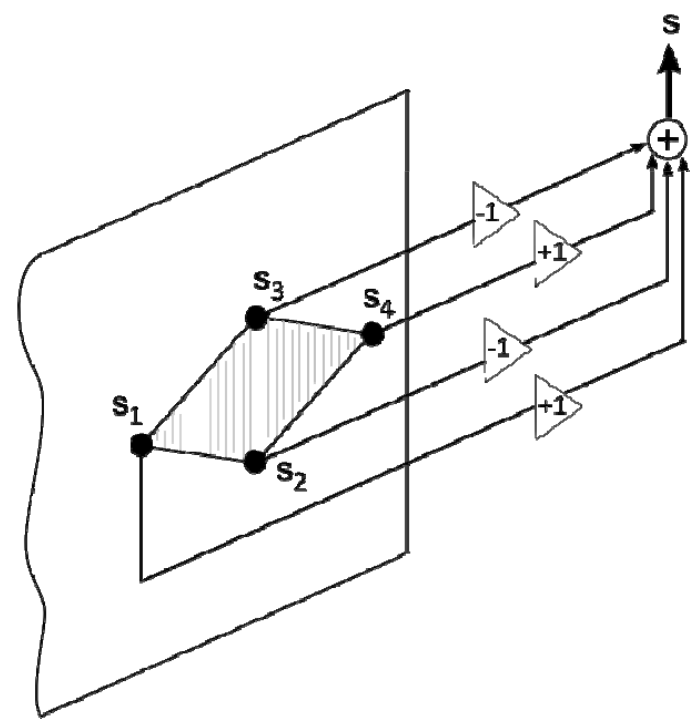

Figure 4: Combination of point sensors that couple to the structural response in the same way as the diamond shaped actuator.

The development of a practical active vibration control system requires more than just matched transducers. It also requires compact electronics, which include signal conditioning, scaling, summing, integration, and amplification. The next section describes the custom circuits designed and built for this application.

\section{ELECTRONICS}

The individual control units each include signal conditioning, control logic, and amplification. Specifically the

This material is declared a work of the U.S. Government and is not subject to copyright protection in the United States. Approved for public release; distribution is unlimited. 
circuits include four 10-32 input connectors used to attach the accelerometers. Since the accelerometers are standard IEPE (Integrated Electronics Piezo Electric) sensors, the first stage provides the required $8 \mathrm{~mA}$ constant current at 28VDC. A highpass filter is then included to strip the DC component from the input signal. The AC signal from each accelerometer is then scaled, summed, and integrated to generate a signal proportional to velocity instead of acceleration. The integrator is implemented as a low-pass filter with a cutoff frequency of 1.5 Hz to avoid DC wind-up. An adjustable gain stage is then included to set the overall circuit gain. The final stage includes two high voltage amplifiers designed to drive the piezoelectric actuator. The transfer function describing the output voltage, $Y$, due to an input on one of the 10-32 connectors, $X$, can be expressed as

$$
Y(j w) / X(j w)=42,700 K j / f
$$

where $f$ is frequency, and $K$ is the adjustable gain, which can be varied from 10 to 60 . The actual circuit response deviates from this simple model below $20 \mathrm{~Hz}$ due to the inclusion of decoupling capacitors, which essentially act as high-pass filters. At frequencies above $20 \mathrm{kHz}$, the response also deviates from this simple model due to limitations associated with the operational amplifiers used in this design.

Due to the simplicity of the control algorithm, it was possible to build the circuit using only analog electronics. This has size and weight benefits since it eliminates the need for anti-aliasing and reconstruction filters, high sample rate $\mathrm{A} / \mathrm{D}$ and $\mathrm{D} / \mathrm{A}$ converters, and a microprocessor or digital signal processor (DSP). The final circuit, shown in Fig. 5 , is $11 \mathrm{~cm}$ (4.3 in) wide, $6.6 \mathrm{~cm}$ (2.6 in) tall, and $2 \mathrm{~cm}(0.8 \mathrm{in})$ deep. The overall weight of the circuit is $54 \mathrm{~g}(0.12 \mathrm{lb})$. The circuit is designed with cascaded stages allowing for maximum flexibility but little integration. In addition, the circuit board includes several components, including conservative use of power bypass capacitors, decoupling capacitors and test points that are useful during the circuit evaluation, but are unnecessary on a production board. Therefore it is estimated that the size and weight of the board could be cut in half by removing unnecessary items and increasing circuit integration.

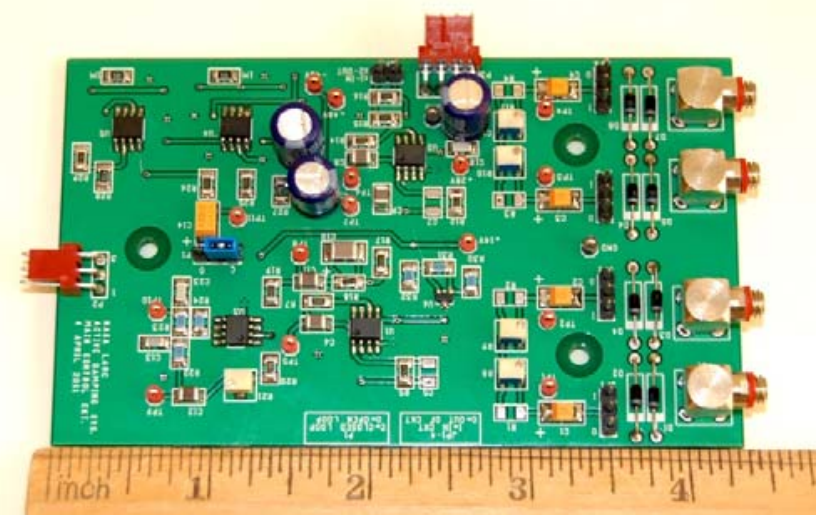

Figure 5: Photograph of the control circuit.
The original goal was to design light-weight compact control circuits that could operate from a standard +28VDC supply common on most aircraft. However piezoelectric actuators, such as the diamond shaped actuator used in this application, require a high drive voltage. Because of the low power requirements of each channel and the comparative efficiencies in DC-DC converters, a separate circuit board was designed to step-up the voltage for all six individual control units. The step-up board, shown in Fig. 6, includes two 100W DC-DC converters, which convert the +28VDC supply to \pm 48 VDC. The fully populated board is $14 \mathrm{~cm}(5.5 \mathrm{in})$ wide by $15.8 \mathrm{~cm}$ (6.2 in) tall by $4.4 \mathrm{~cm}$ (1.7 in) deep and weighs $460 \mathrm{~g}$ $(1.0 \mathrm{lb})$. The size of this board is controlled by the DC-DC converters, which were conservatively chosen with excess reserve capacity and could be replaced with lower power models in subsequent designs.

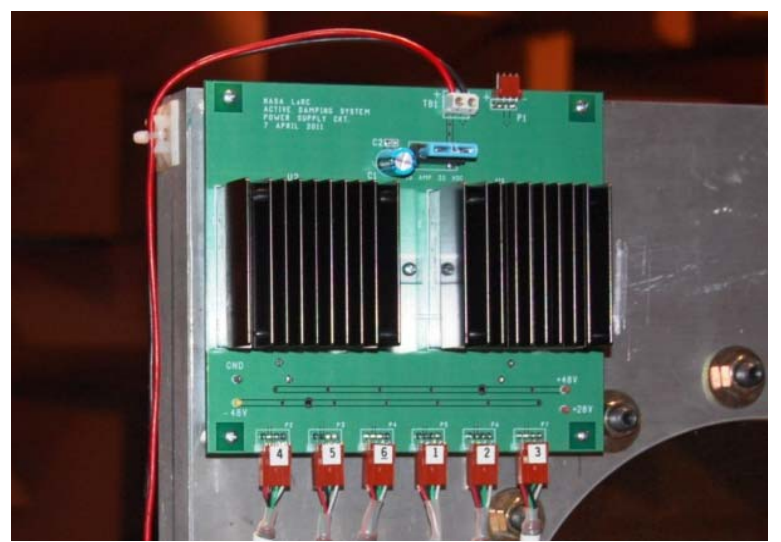

Figure 6: Photograph of the step-up circuit board.

\section{TEST SETUP}

This section describes the test setup used to assess the performance of the active control system. Six control units, each consisting of a diamond shaped actuator, three accelerometers, and a control circuit, were positioned around the perimeter of the Plexiglas window panel shown in Fig. 7. The panel is $4.45 \mathrm{~mm}$ (0.175 in) thick and is sandwiched between two $19 \mathrm{~mm}$ (0.75 in) thick aluminum frames. The frames are held together with fifty bolts torqued to $16.9 \mathrm{~N}-\mathrm{m}$ (150 in-lbs). The outer dimensions of the frame are $90.4 \mathrm{~cm}$ (35.6 in) by $70.9 \mathrm{~cm}$ (27.9 in), while the nominal dimensions of the window are $45.5 \mathrm{~cm}$ (17.9 in) by $65.0 \mathrm{~cm}(25.6 \mathrm{in})$. The overall weight of the window is $1.6 \mathrm{~kg}(3.5 \mathrm{lbs})$.

This material is declared a work of the U.S. Government and is not subject to copyright protection in the United States. Approved for public release; distribution is unlimited. 


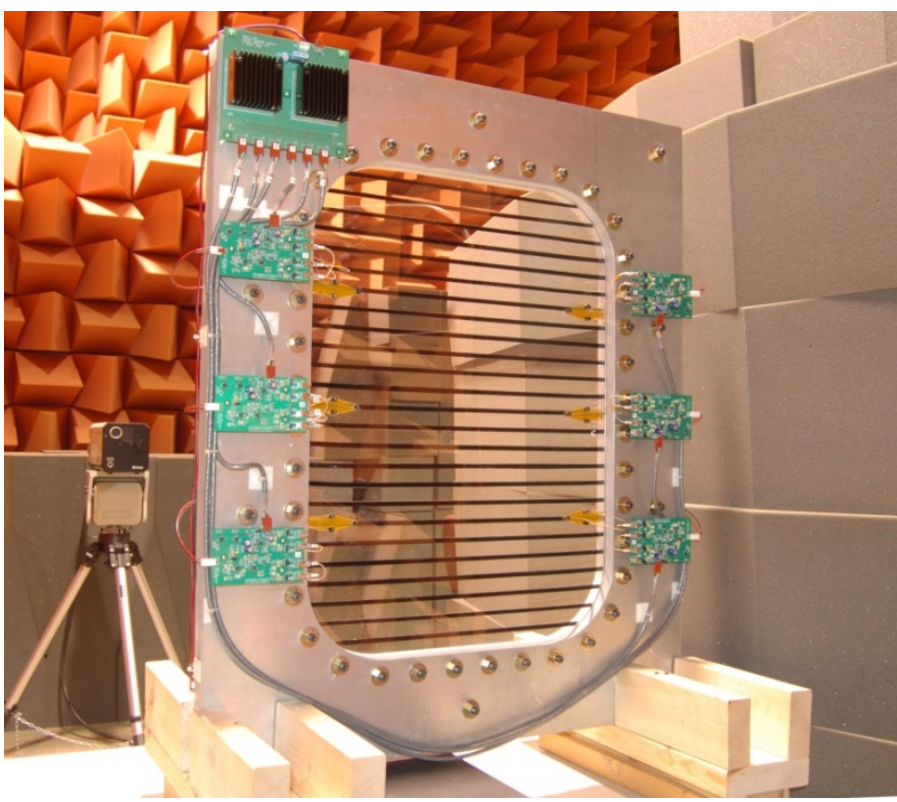

Figure 7: Photograph of the test setup.

The step-up circuit with the two DC-DC converters is attached to the top left hand side of the aluminum frame shown in Fig. 7. For simplicity, a separate power cable is run from this board to each control unit. However in practice the control units could be daisy chained together using a single power cable to save weight. For simplicity, the control unit located on the top right-hand side of the window was not used for control, but was instead used to generate the disturbance. Therefore during the tests, this control circuit was disconnected and the actuator was driven with a broadband pseudorandom excitation.

A Polytec PSV-300 scanning laser vibrometer positioned $2.64 \mathrm{~m}$ (104 in) from the Plexiglas window was used to acquire velocity measurements on an 84 point rectangular grid. Horizontal strips of reflective tape were attached to the window, as seen in Fig. 7, to enable laser measurements on the transparent panel.

A close-up of the transducers is shown in Fig. 8. The diamond shaped piezoelectric actuator weighs just $1.0 \mathrm{~g}$ $(0.0022 \mathrm{lb})$. In this case the base of the actuator is aligned with the edge of the window. Due to the rigidity of the window frame, the relative motion of the base vertex is significantly less than the other vertices. Therefore an accelerometer was not needed at the base, and only three miniature accelerometers, weighing just $0.4 \mathrm{gm}$ each, were positioned around the actuator as shown in Fig. 8. Each actuator was attached to the panel using a two part strain gage epoxy, while the miniature accelerometers were bonded to the structure with a cyanoacrylate adhesive.

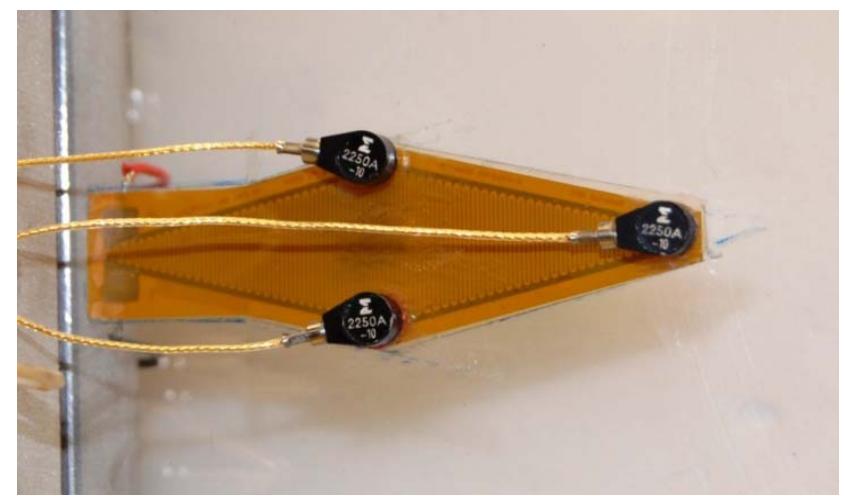

Figure 8: Photograph of a diamond shaped MFC actuator mounted along the perimeter of a Plexiglas panel. Miniature accelerometers are located at three of the four vertices.

As described earlier, the actuators have interdigitated electrodes and a diamond shape. For the MFC actuators used in this study, the optimal ratio of width to length is 0.254 . While this ratio is important, the overall size is not critical and can be selected based on the application. For this application, the actuators are mounted on a $4.45 \mathrm{~mm}(0.175$ in) thick Plexiglas window and the bandwidth of interest extends from $500 \mathrm{~Hz}$ to $3 \mathrm{kHz}$. It was assumed that the distributed actuator would couple to the structure most efficiently when the actuator's length was approximately equal to the bending wavelength in the structure. The bending wavelength in a plate can be approximated as [8]

$$
\lambda=2 \pi\left(\omega^{2} m / D\right)^{-1 / 4}
$$

where $m$ is the mass per unit area, $D=E h^{3} / 12\left(1-v^{2}\right), h$ is the thickness of the plate, $E$ is Young's modulus, and $v$ is Poisson's ratio. In the Plexiglas window, the bending wavelength is approximately $17 \mathrm{~cm}(6.7 \mathrm{in})$ and $6.93 \mathrm{~cm}(2.73 \mathrm{in})$ at $500 \mathrm{~Hz}$ and $3 \mathrm{kHz}$, respectively. Unfortunately large actuators that would efficiently couple to the structure at $500 \mathrm{~Hz}$, would also obstruct a large portion of the window. Therefore the length of the actuator was selected to be $6.35 \mathrm{~cm}(2.5 \mathrm{in})$, or approximately $40 \%$ and $90 \%$ of the bending wavelength at $500 \mathrm{~Hz}$ and $3 \mathrm{kHz}$ respectively. Once the length was specified, the optimal width was calculated to be $1.63 \mathrm{~cm}$ (0.64 in).

The combined weight of a single control unit, which includes the actuator, accelerometers, and control circuit, is currently $56 \mathrm{~g}(0.12 \mathrm{lb})$. However subsequent designs could realistically weigh as little as $29 \mathrm{~g}(0.06 \mathrm{lb})$ by removing unnecessary circuit components and increasing circuit integration. In that case 6 control units would weigh about $10 \%$ of the weight of the bare window. Although these numbers do not include the step-up board or wires, the weight of all necessary system components is still relatively modest.

This material is declared a work of the U.S. Government and is not subject to copyright protection in the United States. Approved for public release; distribution is unlimited. 


\section{RESULTS}

This section describes the performance of the control system. However before comparing open- and closed-loop results, it is beneficial to consider the open-loop frequency response function for a single control unit, as shown in Fig. 9. The frequency response function was acquired by driving the power amplifiers on the control circuit with a broadband random signal while measuring the scaled, summed, and integrated signal from the accelerometers. Therefore this frequency response function includes the dynamics of the structure, the transducers, and the circuit. Due to the relatively small size of the actuator, it does not efficiently couple to the low frequency structural modes, especially below $100 \mathrm{~Hz}$. This explains the poor coherence at low frequencies. The amplitude of the frequency response function is relatively large in the frequency range from about $900 \mathrm{~Hz}$ to $3 \mathrm{kHz}$, which suggests that the actuator was sized correctly for this application. The phase response can be used to determine whether or not the transducers are matched. When the phase response is bounded between \pm 90 degrees, the transducers are matched. In this case, the phase response is bounded through $7.5 \mathrm{kHz}$. The undesirable phase response at higher frequencies could be due to actuator shaping or sensor placement errors.

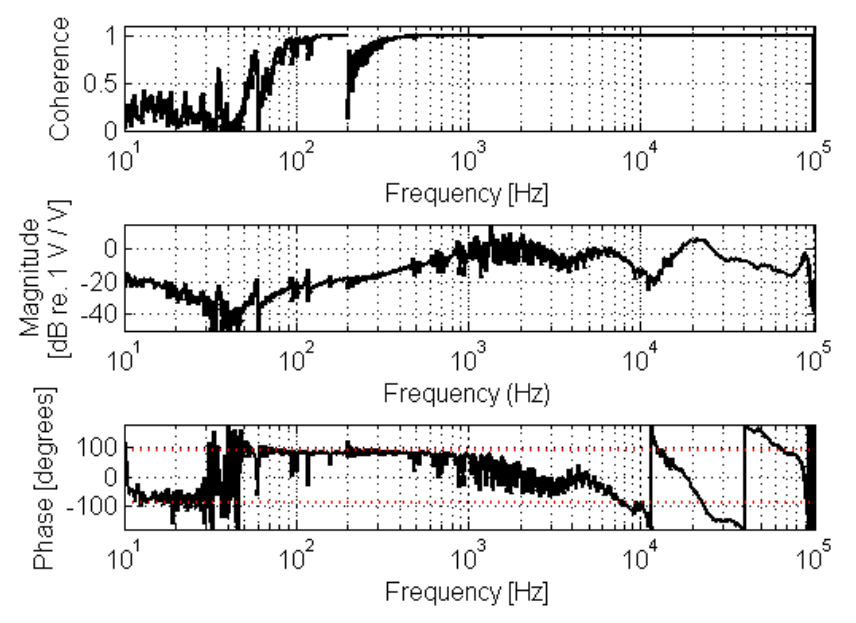

Figure 9: Open loop frequency response function for a representative control unit. The dotted red lines in the phase plot indicate \pm 90 degrees.

Figure 10 shows the Nyquist diagram of the open loop frequency response function. The Nyquist diagram is commonly used to assess the stability and performance of control systems. The performance can be estimated based on the size of the loops in the right half plane (everything to the right of the vertical line running through zero). Larger loops indicate better performance. In contrast the loops in the left half plane mean that the actuator and sensor couple to the structural response out-of-phase. Therefore loops in the left half plane are undesirable. Notice that the large loops in the right half plane correspond to frequencies between $1 \mathrm{kHz}$ and $5 \mathrm{kHz}$. This is the frequency range where the transducers couple most efficiently to the structure. The small loops in the left half plane correspond to frequencies above $5 \mathrm{kHz}$. The stability of a single control unit can be inferred from the Nyquist stability criterion. If the plant and controller are both stable, then the Nyquist stability definition states that the closed loop system will be stable if and only if the polar plot of the open-loop frequency response does not encircle the $(-1,0)$ point as the frequency varies from $-\infty$ to $\infty$ [9]. Based on the Nyquist diagram, it is clear that this control unit will be stable when the loop is closed. However just because each individual control loop is stable, does not mean that all 5 control units will be stable when implemented together. To evaluate stability of the entire set of control loops, the generalized Nyquist stability criterion can be used [9]. However in this application it is relatively easy to set the control gain on each circuit and then check closed-loop stability. Therefore the generalized Nyquist criterion was not used. It is interesting to note that when the gains were increased to the point where the system went unstable, the response was dominated by a high amplitude peak around $40 \mathrm{kHz}$, which denoted a limit cycle. As long as the system was at least marginally stable, very little control spillover occurred within the audible frequency range.

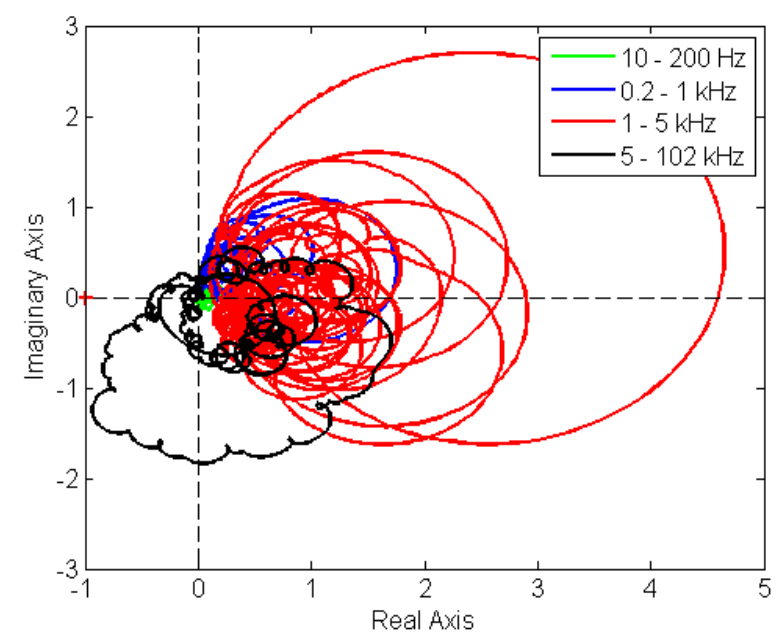

Figure 10: Nyquist diagram of the open loop frequency response function from $10 \mathrm{~Hz}$ to $102 \mathrm{kHz}$.

The next step was to study the damping of the window as a function of control gain, and also as a function of the number of control units. The impulse response decay method (IRDM) $[10,11]$ was used to estimate the total loss factor of the window. The total loss factor includes all energy loss mechanisms, including structural damping, sound radiation, and transmission to other components. While it is possible for the total loss factor to be much larger than the damping loss factor, in this case energy losses due to sound radiation are expected to be small between $500 \mathrm{~Hz}$ and $3 \mathrm{kHz}$ since the coincidence frequency of the window occurs around $8 \mathrm{kHz}$. In addition, the

This material is declared a work of the U.S. Government and is not subject to copyright protection in the United States. Approved for public release; distribution is unlimited. 
large impedance mismatch between the aluminum frame and the Plexiglas window should prevent significant energy flow through the boundary of the window. Therefore the total loss factor estimate from IRDM should be a good approximation of the damping loss factor of the window.

The impulse response decay method provides $1 / 3$-octave band estimates of the loss factor from broadband frequency responses. In this case, eighty-four unique frequency responses were acquired while exciting the window with one of the diamond shaped actuators. The frequency response functions are first windowed to obtain the band-limited response within a specific 1/3-octave band. The inverse Fourier transform is then used to compute the impulse response from each 1/3-octave band windowed-FRF. The normalized Schroeder decay curve is calculated from the impulse response and then a linear regression is used to determine the loss factor.

Figure 11 shows damping estimates for the window in the 1/3-octave bands from $125 \mathrm{~Hz}$ through $10 \mathrm{kHz}$. The vertical dashed red lines bound the frequency range of interest (i.e. 500 $\mathrm{Hz}$ through $3 \mathrm{kHz}$ ). In this case, the same control gain is used for all 5 control units. The figure shows that the control units increase the damping loss factor from $1 \%$ to $1.5 \%$ in the frequency range of interest. However there is little change in the damping loss factor as the control gain is varied from 20 to 40. Previous work suggests that there should be an optimal gain, which maximizes the damping of the structure [2]. Extremely large control gains are not necessarily desirable since the controller would essentially pin the structure, resulting in new lightly damped resonances. Future studies will consider a larger range of control gains in an effort to determine the optimal value. It could also be possible to implement an automated gain tuning algorithm within the control circuit. A recent study by Zilletti et al. [12] suggests that an adaptive approach could be used to tune the gain and optimize the power absorbed by each control unit.

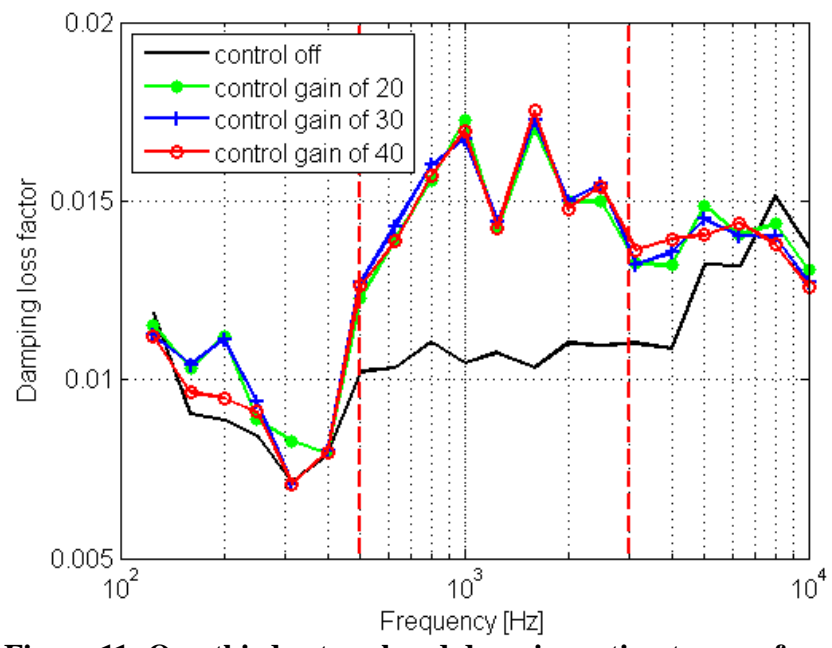

Figure 11: One-third octave band damping estimates as a function of control gain.
Figure 12 shows how the damping of the window changes as control units are added. For this investigation a fixed gain of 30 was used on all control units. The first thing to note is that damping increases as control units are added. The relatively small change in damping with the addition of the $5^{\text {th }}$ control unit deserves further attention. Separate tests demonstrated that some control units are more effective than others. It is possible that the $5^{\text {th }}$ control unit is simply not effective due to its position on the window. It is also possible that additional control units provide diminishing returns. This issue will be addressed in subsequent tests.

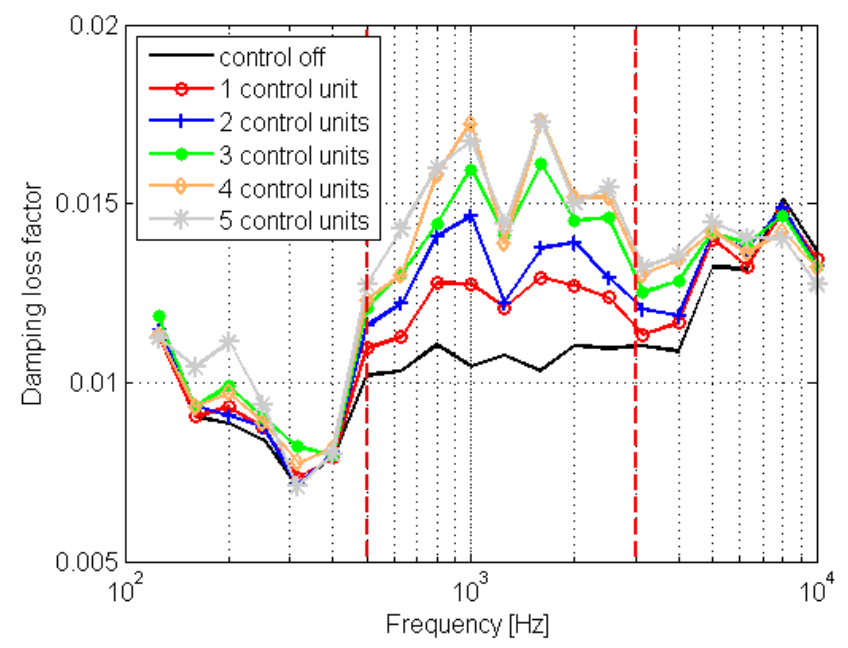

Figure 12: One-third octave band damping estimates as a function of the number of control units.

The performance of the control units can also be evaluated in terms of the spatially averaged velocity of the window, as shown in Fig. 13. Once again, a fixed gain of 30 was used for all 5 control units. The difference between the open-loop (black line) and closed-loop (red line) response is significant within the bandwidth of interest. The control system reduces individual resonance peaks by as much as $10 \mathrm{~dB}$ and achieves a $2.4 \mathrm{~dB}$ integrated reduction from $500 \mathrm{~Hz}$ to $3 \mathrm{kHz}$. In addition, there is negligible control spillover through $10 \mathrm{kHz}$.

This material is declared a work of the U.S. Government and is not subject to copyright protection in the United States. Approved for public release; distribution is unlimited. 


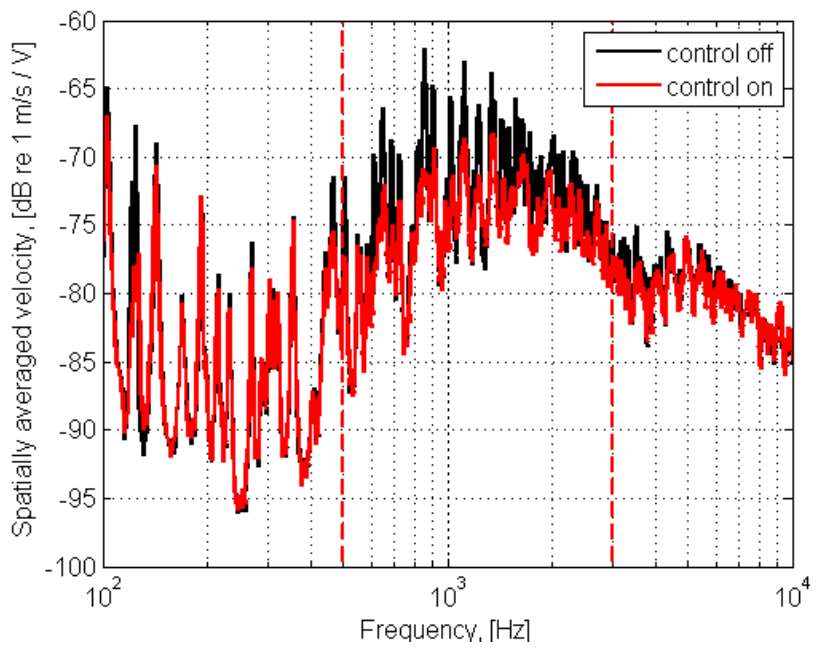

Figure 13: Spatially averaged velocity normalized by the drive signal: control off (solid black line), and control on (solid red line).

\section{CONCLUDING REMARKS}

This paper describes the development of a compact active vibration control system that is well suited for use on lightly damped flexible structures. The system is composed of a diamond shaped piezoelectric patch actuator with interdigitated electrodes. This actuator couples to the structure in the same way as a collection of point forces. Therefore point sensors can be combined with the actuator to form a matched transducer pair. A compact control circuit has also been designed and built, which combines signal conditioning, control logic, and power electronics on a single board.

Five independent control units were installed and tested on a Plexiglas panel, representative of a helicopter window. The control units increased the structural damping from about $1 \%$ to $1.5 \%$. This reduced the spatially averaged velocity of the panel by $10 \mathrm{~dB}$ at individual resonances and achieved an integrated reduction of $2.4 \mathrm{~dB}$ from $500 \mathrm{~Hz}$ through $3 \mathrm{kHz}$. In addition, the control units generated negligible spillover through $10 \mathrm{kHz}$.

Future work will focus on identifying the optimal control gain for each control unit. This could be done manually or automated within the control circuit. Additional modeling would also be helpful to predict the performance of each control unit based on its location on the structure. The model could also be used to assess the additive effect of additional control units.

\section{ACKNOWLEDGEMENTS}

The authors gratefully acknowledge the support of John Callahan who laid out and populated the circuit boards.

\section{REFERENCES}

[1] Buehrle, R. D., Klos, J., and Gibbs, G. P., 2004, "Damped windows for aircraft interior noise control," Proc. Noise-Con 2004, Baltimore, Maryland.
[2] Gardonio, P., and Elliott, S. J., 2005, "Smart panels with velocity feedback control systems using triangularly shaped strain actuators," J. Acoustical Society of America, 117(4), pp. 2046-2064.

[3] Aoki, Y., Gardonio, P., and Elliott, S. J., 2008, "Rectangular plate with velocity feedback loops using triangularly shaped piezoceramic actuators: Experimental control performance," 123(3), pp. 1421-1426.

[4] Gardonio, P., Aoki, Y., and Elliott, S. J., 2009, "Stability and control performance of decentralized velocity feedback loops using triangular actuators," Proc. Active 2009, Ottawa, Canada.

[5] Gardonio, P., Bianchi, E., and Elliott, S. J., 2004, "Smart panel with multiple decentralized units for the control of sound transmission. Part I: theoretical predictions," J. Sound and Vibration, 274, pp. 163-192.

[6] Schiller, N. H., Cabell, R. H., Quinones, J. D., and Wier, N. C., 2010, "Active damping using distributed anisotropic actuators," Proc. ASME IMECE 2010, Vancouver, Canada.

[7] Sullivan, J. M., Hubbard, J. E., and Burke, S. E., 1996, "Modeling approach for two-dimensional distributed transducers of arbitrary spatial distribution," J. Acoustical Society of America, 99(5), pp. 2965-2974.

[8] Fahy, F., and Gardonio, P., 2007, Sound and Structural Vibration $2^{\text {nd }}$ Edition. Academic Press, Oxford, UK, Chap 1.

[9] Elliott, S. J., 2001, Signal Processing for Active Control, Academic Press, London, UK, Chap. 6.

[10] Bloss, B. C., and Rao, M. D., 2005, "Estimation of frequency-averaged loss factors by the power injection and the impulse response decay methods," J. Acoustical Society of America, 117(1), pp. 240-249.

[11] Cabell, R., Schiller, N., Allen, A., and Moeller, M., "Loss factor estimation using the impulse response decay method on a stiffened structure," Proc. Inter-Noise 2009, Ottawa, Canada. [12] Zilletti, M., Elliott, S. J., and Gardonio, P., 2010, "Selftuning control systems of decentralised velocity feedback," J. Sound and Vibration, 329(14), pp. 2738-2750.

This material is declared a work of the U.S. Government and is not subject to copyright protection in the United States. Approved for public release; distribution is unlimited. 
This material is declared a work of the U.S. Government and is not subject to copyright protection in the United States. Approved for public release; distribution is unlimited. 